\title{
Incidentally Discovered
}

\section{Adenocarcinoma in situ of the Appendix in a Young Woman}

\author{
Noriaki Koizumi Yasutoshi Murayama Yoshiaki Kuriu \\ Masayoshi Nakanishi Kazuma Okamoto Yukihito Kokuba \\ Eigo Otsuji
}

Division of Digestive Surgery, Department of Surgery, Kyoto Prefectural

University of Medicine, Kyoto, Japan

\section{Key Words}

Early appendiceal cancer - Appendiceal adenocarcinoma - Surgical treatment .

Histopathological examination

\begin{abstract}
Primary appendiceal adenocarcinoma is an infrequent disease. This report presents a rare case of incidentally discovered carcinoma in situ of the appendix. A 35-year-old parturient female simultaneously underwent appendectomy and oophorectomy due to an ovarian abscess that adhered to the appendix during cesarean section. Although her excised appendix showed no apparent tumorous lesion, histopathological examination revealed carcinoma in situ in the excised appendix. She underwent additional right hemicolectomy a few days later due to the possibility of a positive surgical margin. Histopathological examination detected no malignant cells in the resected specimen. She was discharged without any complications and has since remained healthy. Appendiceal adenocarcinoma is generally considered to be difficult to diagnose during the early stage because it seldom shows any specific findings. This results in a poor prognosis. Histopathological examination is not always conducted for appendices resected during other surgery. However, the current study suggests that a careful routine histopathological examination of excised appendix, as well as careful preoperative examination and detailed intraperitoneal inspection during surgery, is indeed important to detect occult appendiceal tumors.
\end{abstract}

\section{Introduction}

Primary adenocarcinoma arising from the vermiform appendix is an infrequent disease and detection of these tumors at an early stage is particularly rare $[1,2]$. Appendiceal tumors seldom show any specific symptoms and little is known about 
their specific findings on examination, thus it is difficult to preoperatively diagnose them. The preoperative detection rate of appendiceal tumors is low, ranging from 6.6 to $25 \%$, and no sufficiently accurate diagnostic modalities have so far been developed [3]. Therefore, many malignant appendiceal tumors, including appendiceal adenocarcinoma, are diagnosed at an advanced stage, thus resulting in their poor prognosis compared to usual colon cancers $[2,4]$. In order to improve the prognosis of such patients, it is indeed important to precisely detect appendiceal tumors at an early stage.

This report presents the rare case of a young female with carcinoma in situ of the vermiform appendix incidentally discovered following gynecologic operation, with a review of the pertinent literature. She had just undergone appendectomy together with oophorectomy. Although she had shown no apparent symptoms related to appendiceal carcinoma preoperatively, and similarly we had never detected any suggestive findings of it even during the previous operation, a detailed histopathological examination revealed the presence of carcinoma in situ within the excised appendix.

We would therefore like to emphasize the significance of performing careful and detailed postoperative histopathological examinations in order to identify occult appendiceal tumors in a timely manner, as well as performing routine preoperative examinations and intraoperative inspections. A precise diagnosis can lead us to carry out adequate and timely surgical procedures, and accordingly improve the prognosis of patients with appendiceal tumors.

\section{Case Report}

A 35-year-old parturient female was referred to the hospital and underwent cesarean section in November 2008. During operation, she was diagnosed to be complicated with a right ovarian abscess. The surgeons simultaneously performed right oophorectomy with appendectomy because her ovary tightly adhered to the vermiform appendix. Macroscopic observation of the resected appendix showed only inflammatory changes, but no apparent tumorous lesion (fig. 1). However, histopathological examination revealed well-differentiated adenocarcinoma confined to the mucosa. Dysplastic epithelial cells were found fragmentally in the proximal side of the resected appendix, and well-differentiated adenocarcinoma in situ was partially observed (fig. 2a, b). Staining with MIB1 and p53 was stronger in the dysplastic lesion in comparison to the neighboring normal epithelium (fig. 2c, d). Dysplastic epithelium was found even at the surgical margin although the degree was relatively low.

She was therefore re-admitted to the hospital for further examination, based on these histopathological findings. Blood tests showed no major abnormality. The CEA and CA19-9 tumor markers were both within normal limits. Colonoscopy showed an elevated lesion around the appendiceal orifice resulting from the appendectomy, but no malignant findings were demonstrated by biopsy (fig. 3). Abdominal contrast-enhanced computed tomography (CT) showed neither any apparent mass lesion nor swollen lymph nodes around the ileocecal area.

Although there was no evidence of any apparent residual cancers, an additional operation was performed due to the suspicion of a positive surgical margin. The second operation revealed the stump of the appendix to demonstrate an induration. No apparent swollen lymph node was observed. The surgery was initially planned to perform partial resection of the cecum, however that seemed to have the potential to cause passage obstruction due to postoperative deformity. As a result, o nly a right hemicolectomy was performed without any complications. Histopathological examination revealed no apparent residual atypical cells in the additionally resected specimen. Her postoperative course was uneventful and she was discharged 9 days after surgery. She has since remained healthy and there have been no signs of recurrence. 


\section{Discussion}

The incidence of primary appendiceal cancer other than carcinoid tumor is estimated to be $<0.5 \%$ of all gastrointestinal neoplasms and $0.1-0.8 \%$ of appendectomy specimens $[3,5,6]$. Primary appendiceal adenocarcinoma is generally classified into two main types: cystic and colonic type. Whereas cystic-type adenocarcinoma is much more common and accounts for about two-thirds of all appendiceal tumors, colonic-type adenocarcinoma is rare [4]. Early-stage colonic-type adenocarcinoma, such as in the current case, is quite rare. Only 32 cases, including the present case, of early colonic-type adenocarcinoma have been reported to date in the Japanese literature [7]. The prognosis of appendiceal adenocarcinoma is said to be poor in comparison to colon cancers arising from other portions of the intestine, the overall 5 -year survival rate of appendiceal adenocarcinoma being 49-64\% [1, 4, 8]. This is mainly because the vast majority of appendiceal adenocarcinomas is diagnosed only after disease progression. The tumor is difficult to detect at an early stage because it shows few specific symptoms or findings on examination. Most colonic-type appendiceal adenocarcinomas are discovered incidentally as a result of acute appendicitis caused by luminal obstruction associated with tumor progression. In addition, relatively rapid progression due to the anatomical features of the appendix is also partially responsible for the poor prognosis. The appendix contains abundant lymphatic tissues and a relatively thin layer, so metastasization to regional lymph nodes and/or dissemination to the peritoneal cavity is likely [1]. Early detection is a key point to achieve improved therapeutic results of appendiceal adenocarcinoma.

It is difficult to find appendiceal tumors by routine colonoscopy, whereas some appendiceal tumors show a characteristic colonoscopy finding called 'volcano sign', consisting of an enlarged protruding orifice of the appendix [9]. However, colonoscopy does not allow a qualitative diagnosis without a sufficient biopsy specimen, and therefore its diagnostic power for appendiceal tumors is still unsatisfactory. In fact, Trivedi et al. [6] reported that preoperative biopsies showed a malignant diagnosis of only $3.1 \%$. Meanwhile, ${ }^{18} \mathrm{~F}$-fluorodeoxyglucose positron emission tomography (FDG-PET) is now widely used as a new diagnostic modality. While there are some false-positive cases demonstrated by PET-CT [10], some cases of appendiceal adenocarcinoma are preoperatively diagnosed by PET-CT [11]. The efficacy of PET-CT for the early diagnosis of appendiceal adenocarcinoma is still controversial, but it may be a helpful diagnostic modality when used in combination with other types of examinations, such as colonoscopy.

Recurrence after surgery is significantly reduced by right hemicolectomy in comparison to appendectomy alone. The 5 -year survival rate improves to $20 \%$ after appendectomy alone, and to $63 \%$ after right hemicolectomy [12]. Therefore, the standard surgical procedure for appendiceal adenocarcinoma is right hemicolectomy with appropriate lymph node dissection, the same as for common colorectal cancers. Appendectomy is regarded as an optimal and sufficient procedure only in lesions confined to the mucosa with a negative surgical margin. Although colonoscopy and biopsy showed no apparent abnormal findings, residual atypical cells were not microscopically deniable because histopathological examination of the surgical margin of the appendix revealed atypical cells. Therefore, radical right hemicolectomy was performed in this case. Although partial resection of the cecum was a possible less 
invasive procedure, it is usually not a standard procedure and may cause deformity of the cecum, thus resulting in passage obstruction.

Colonoscopy in association with secondary operation is one of the essential preoperative examinations besides abdominal CT. It is also important to carefully check for the presence or absence of atypical cells around the appendiceal orifice and to rule out any other synchronous colorectal lesions because colorectal cancer is often multifocal [6]. Long-term follow-up by colonoscopy is also recommended, especially in juvenile patients with colonic-type appendiceal adenocarcinoma, since they appear to have a higher risk to develop another colorectal cancer.

Some reports recommend limiting routine histopathological examination for surgical specimens of limited or no clinical value to reduce medical costs $[13,14]$. However, even though gross inspection may demonstrate no tumorous lesion, there are some cases in whom histopathological examination does indeed demonstrate abnormal findings, as in the current case. Therefore, histopathological examination must always be done after not only appendectomy for acute appendicitis, but also after combined resection following another operation [15]. Therefore, careful preoperative examination, detailed intraperitoneal inspection during surgery, and especially careful routine histopathological examinations of the excised appendix are important to detect the presence of occult appendiceal tumors. 

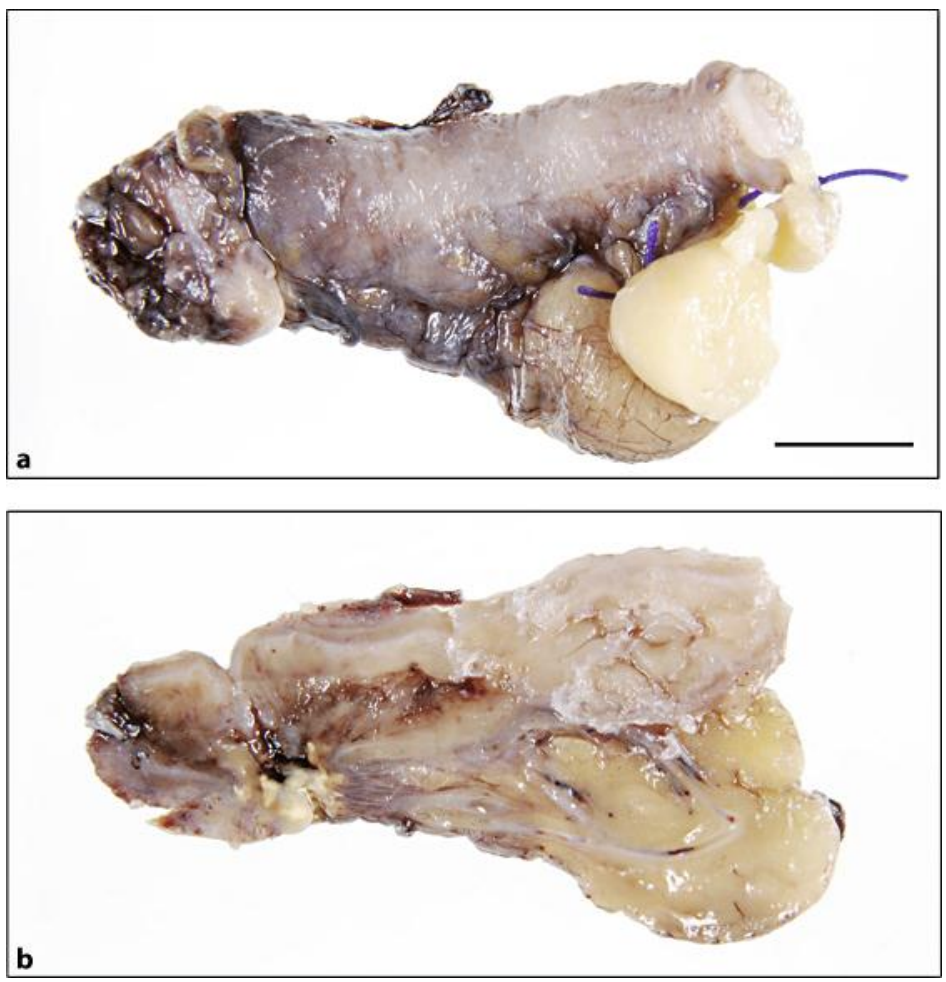

Fig. 1. a The excised appendectomy specimen showed slight swelling due to inflammatory change, but no neoplastic changes were observed on the serosal surface. $\mathbf{b}$ The sectioned surface of an excised specimen showed no apparent neoplastic lesions macroscopically. Scale bars $=10 \mathrm{~mm}$. 


\begin{tabular}{r|l|l|l}
$\begin{array}{r}\text { Case Reports in } \\
\text { Gastroenterology }\end{array}$ & $\begin{array}{l}\text { Case Rep Gastroenterol 2012;6:726-733 } \\
\text { DOI: 10.1159/000345805 }\end{array}$ & $\begin{array}{l}\text { Published online: } \\
\text { November 22, 2012 }\end{array}$ & $\begin{array}{l}\text { C 2012 S. Karger AG, Basel } \\
\text { ISSN 1662-0631 } \\
\text { www.karger.com/crg }\end{array}$ \\
\hline
\end{tabular}
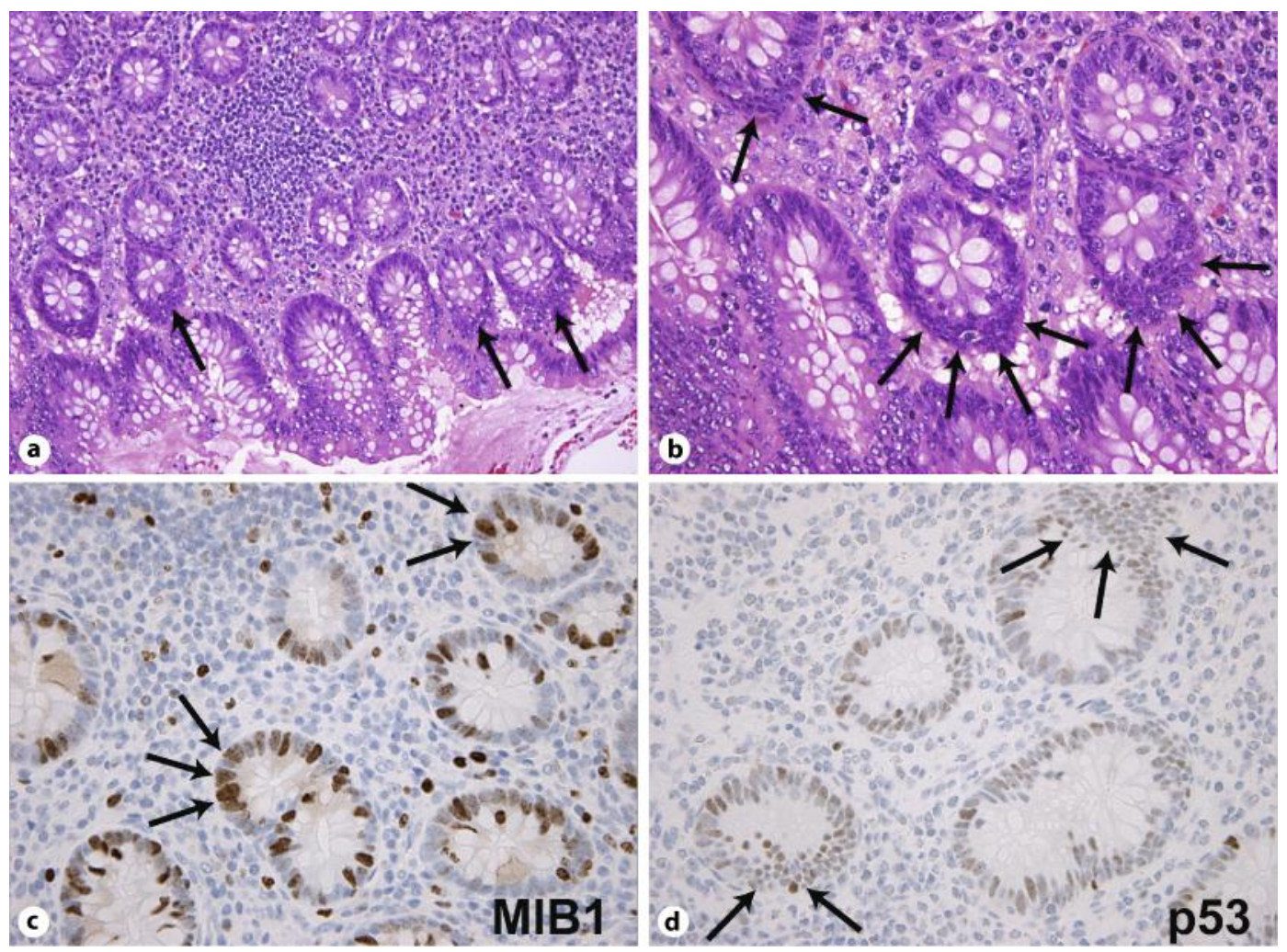

Fig. 2. a, b Histopathological findings sporadically showed dysplastic epithelial cells with nuclear atypia and disarranged cellular polarities (arrows), which were histopathologically defined as well-differentiated adenocarcinoma in situ (hematoxylin-eosin staining, a $\times 200, \mathbf{b} \times 400$ ).

c Immunohistochemical staining with MIB1 showed increased staining properties of atypical epithelial cells (arrows). MIB1 labeling index was increased up to 24\%, whereas epithelial cells of the neighboring normal gland showed low MIB1 labeling index of $<10 \%(\times 400)$. d Immunohistochemical staining with p53 showed increased staining properties mainly at a part of glands where the cellular polarities of epithelial cells were disarranged (arrows) $(\times 400)$. p53 positivity was increased up to $63 \%$. 


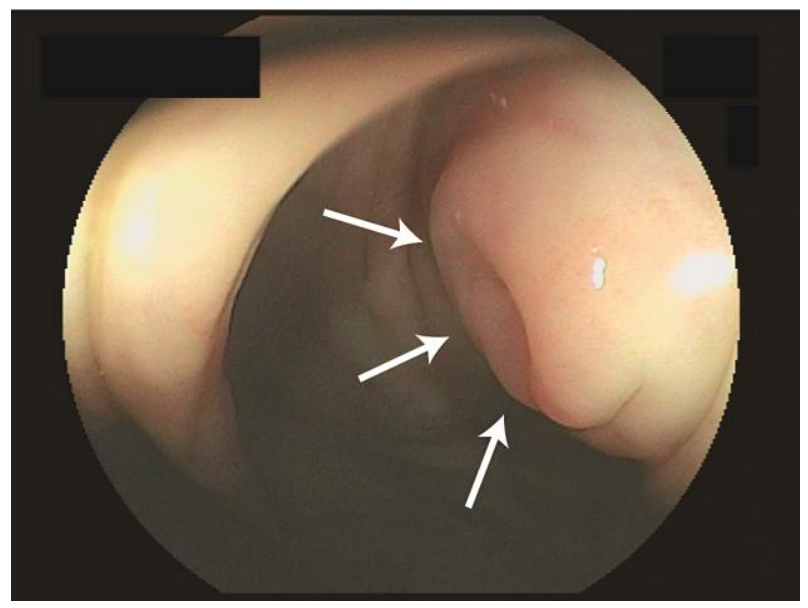

Fig. 3. Colonoscopy prior to the secondary operation showed an elevated lesion around the appendiceal orifice due to previous appendectomy (arrows). However, the mucosal surface was intact, and no surface abnormalities suggestive of epithelial lesions were observed.

\section{References}

1 Benedix F, Reimer A, Gastinger I, Mroczkowski P, Lippert H, Kube R: Primary appendiceal carcinoma epidemiology, surgery and survival: results of a German multi-center study. Eur J Surg Oncol 2010;36:763-771.

2 Lee WS, Choi ST, Lee JN, Kim KK, Park YH, Baek JH: A retrospective clinicopathological analysis of appendiceal tumors from 3,744 appendectomies: a single-institution study. Int J Colorectal Dis 2011;26:617-621.

-3 Oya S, Miyata K, Yuasa N, Takeuchi E, Goto Y, Miyake H, Nagasawa K, Kobayashi Y, Ito T, Ito M: Early carcinoma of the appendix vermiformis. Dig Endosc 2009;21:53-55.

-4 Ko YH, Park SH, Jung CK, Won HS, Hong SH, Park JC, Roh SY, Woo IS, Kang JH, Hong YS, Byun JH: Clinical characteristics and prognostic factors for primary appendiceal carcinoma. Asia Pac J Clin Oncol 2010;6:19-27.

5 Ozakyol AH, Saricam T, Kabukcuoglu S, Caga T, Erenoglu E: Primary appendiceal adenocarcinoma. Am J Clin Oncol 1999;22:458-459.

-6 Trivedi AN, Levine EA, Mishra G: Adenocarcinoma of the appendix is rarely detected by colonoscopy. J Gastrointest Surg 2009;13:668-675.

7 Suzuki S: Two cases of early vermiform appendix adenocarcinoma and a review of 42 cases in Japan. J Jpn Coll Surg 2011;36:36-39.

>8 Walters KC, Paton BL, Schmelzer TS, Gersin KS, Iannitti DA, Kercher KW, Heniford BT: Treatment of appendiceal adenocarcinoma in the United States: penetration and outcomes of current guidelines. Am Surg 2008;74:1066-1068.

9 Bruna T, Gineston JL, Lamblin G, Viard P, Auge MC: Endoscopic diagnosis and treatment of an appendiceal mucocele: under the sign of volcano. Gastroenterol Clin Biol 1995;19:229-230.

10 Ogawa S, Itabashi M, Kameoka S: Significance of FDG-PET in identification of diseases of the appendix based on experience of two cases falsely positive for FDG accumulation. Case Rep Gastroenterol 2009;3:125-130.

11 Lu SJ, Osmany S, Khoo JB, Ng DC: Synchronous adenocarcinoma of the appendix demonstrated on F-18 FDG PET/CT. Clin Nucl Med 2010;35:256-257. 
12 Hesketh KT: The management of primary adenocarcinoma of the vermiform appendix. Gut 1963;4: 158-168.

13 Cross SS, Stone JL: Proactive management of histopathology workloads: analysis of the UK Royal College of Pathologists' recommendations on specimens of limited or no clinical value on the workload of a teaching hospital gastrointestinal pathology service. J Clin Pathol 2002;55:850-852.

14 Fitzgibbons P, Cleary K: CAP offers recommendations on selecting surgical specimens for examination CAP Today 1996;10:40.

-15 Swank HA, Eshuis EJ, Ubbink DT, Bemelman WA: Is routine histopathological examination of appendectomy specimens useful? A systematic review of the literature. Colorectal Dis 2011;13: $1214-1221$. 